\section{Steroids, atosiban and pulmonary} oedema: are or may be a cause?

We read with interest the letter from Nijs et al. ${ }^{1}$ describing a case of pulmonary oedema in a preeclamptic twin pregnancy in which steroid and atosiban tocolytic therapy were thought to be contributory. It is a useful reminder of potential complications in such high-risk patients and we agree that careful monitoring is essential to detect deterioration early. However, there are several points in the article with which we have issue.

In this case it is difficult to be certain that the combination of steroids and atosiban is a significant contributor to pulmonary oedema, due to the presence of many other well-recognised risk factors. Preeclampsia can precipitate pulmonary oedema, and the associated greater afterload would increase this risk further in mitral valve insufficiency. ${ }^{2}$ Twin pregnancy is also known to increase the risk of pulmonary oedema with atosiban, as noted in the summary of product characteristics (SmPC). ${ }^{3}$

Other risk factors potentially present in this case were not mentioned. We would be interested to know if the fluid balance was recorded prior to onset of dyspnoea, as excessive fluid intake may precipitate pulmonary oedema, especially in preeclampsia. ${ }^{3}$ Did the patient receive anti-hypertensive or additional tocolytic treatment, as co-administration of calcium channel blockers with atosiban is a recognised risk factor for pulmonary oedema? ${ }^{2}$ The evidence referenced for the effect of combined steroid and tocolytic therapy causing pulmonary oedema is a retrospective study that does not include atosiban as one of the tocolytics. ${ }^{4}$ Given the multitude of other risk factors it would seem difficult to reliably identify the steroid and atosiban combination as a significant contributor in this case.

We also disagree with the statement "Because of its molecular structure it has, at least theoretically, an affinity for vasopressin receptors so inhibition of anti-diuretic effects may cause congestive cardiac failure and hypertension.$^{1}$ Antagonism of the effect of oxytocin at vasopressin receptors would be expected to result in hypotension and diuresis. The SmPC for atosiban makes no mention of hypertension but does note the risk of hypotension. ${ }^{3}$ The Fernandez et al. $^{5}$ reference for this assertion provides no evidence that atosiban causes hypertension or cardiac failure, indeed it references a different article ${ }^{6}$ which also contains no evidence for this effect. This same issue has been raised previously in this journal in response to a similar case.

Balancing the well-proven benefits of antenatal steroid therapy for premature infants ${ }^{8}$ against the uncertain effect of combined steroid and atosiban therapy, it would seem prudent to suggest that combining steroids and atosiban, for the management of preterm labour, "may be a risk factor for pulmonary oedema, rather than "is a risk factor.

J. Guy, E. O'Kane, A. Eggleton

Department of Anaesthesia, Ulster Hospital, Dundonald Northern Ireland, United Kingdom

E-mail address: andrew.eggleton@setrust.hscni.net

\section{References}

1. Nijs K, Nulens K, Van De Velde M, Stessel B. The combination of corticosteroid and tocolytic therapy in a preeclamptic patient is a risk factor for the development of acute pulmonary oedema. Int Obstet Anesth 2018:34:113-4.

2. Dennis A, Solnordal C. Acute pulmonary oedema in pregnant women. Anaesthesia 2012;67:646-59.

3. https://www.medicines.org.uk/emc/product/3262 Accessed January 2018.

4. Ogunyemi D. Risk factors for acute pulmonary edema in preterm delivery. Eur J Obstet Gynecol Reprod Biol 2007;133:143-7.

5. Fernandez A, Dom nguez D, Delgado L. Severe non-cardiogenic pulmonary oedema secondary to atosiban and steroids. Int $J$ Obstet Anesth 2011:20:189-90.

6. Afschar P, Scholl W, Bader A, Bauer M, Winter R. A prospective randomised trial of atosiban versus hexoprenaline for acute tocolysis and intrauterine resuscitation. BJOG 2004:111:316-8.

7. Wright G, Levy D. Atosiban and non-cardiogenic pulmonary oedema. Int J Obstet Anesth 2012;21:98-102.

8. Roberts D, Brown J, Medley N, Dalziel S. Antenatal corticosteroids for accelerating fetal lung maturation for women at risk of preterm birth. Cochrane Database Syst Rev 2017:3:CD004454.

0959-289X/\$ - see front matter

Crown Copyright 2018 Published by Elsevier Ltd. All rights reserved. https://doi.org/10.1016/j.ijoa.2018.03.004

\section{In reply}

We thank Guy et al. ${ }^{1}$ for their interesting comments regarding the case we reported, describing the development of acute pulmonary oedema in a patient with preeclampsia. $^{2}$ They make a number of valid points.

We agree that it is difficult to be sure whether the combination of steroids and atosiban was a significant factor in the development of pulmonary oedema in our patient, due to the presence of other risk factors. ${ }^{3}$ We acknowledge that multiple risk factors can be related to the development of pulmonary oedema in a patient such as ours, as was stated in the article. ${ }^{2}$ Both transthoracic echocardiography and transoesophageal echocardiography were conducted and only slight mitral valve insufficiency was found, thereby excluding a potentially substantial causal factor. We agree that even a small increase in mitral valve insufficiency can be a "supplementary risk factor in the development of acute pulmonary oedema. 
The other potential risk factors mentioned by Guy et al. ${ }^{1}$ are reasonable concerns, given the limited amount of information they received. However not all of these risk factors were present in our patient, who had an initial diagnosis of cervical shortening and for mobilisation reasons stayed on the maternity ward, with only intravenous (IV) access via an "IV clear connector . Her fluid intake therefore was purely oral. Also, the appearance and severity of pre-eclampsia evolved rapidly, such that due to the rapid onset of respiratory distress, antihypertensive treatment was not started before the caesarean section. In our correspondence, ${ }^{2}$ we simply acknowledged that the combination of corticosteroid and tocolytic therapy in a preeclamptic patient is a risk factor for the development of acute pulmonary oedema. At no time did we state that it was the only risk factor in this complicated clinical case, where multiple comorbidities were present.

The summary of product characteristics (SmPC) for atosiban ${ }^{4}$ contains a warning to be careful if atosiban is administered to pregnant women with twins. Respiratory events, such as dyspnoea and pulmonary oedema, particularly in women with multiple pregnancy, have been reported. ${ }^{4}$ Atosiban is said to be contraindicated in "Eclampsia and severe pre-eclampsia requiring delivery in the SmPC. ${ }^{4}$ However an obstetrician will sometimes take the risk of using atosiban in these circumstances, in what they consider to be in the best interest of the patient and fetus. By reviewing the SmPC of atosiban we also found the statement that "As an antagonist of oxytocin, atosiban may theoretically facilitate uterine relaxation and postpartum bleeding. Therefore blood loss after delivery should be monitored. ${ }^{4}$ That being said, regular arterial blood gas analyses are a superior method for the frequent evaluation of anaemia in patients.

Guy et al. ${ }^{1}$ disagreed with us about the effect of atosiban on the maternal blood pressure. However in the literature, multiple sources state that it has a possible hypertensive effect. ${ }^{5,6}$

Last, but not least, we absolutely agree with Guy et al. ${ }^{1}$ that the benefits of antenatal steroid therapy and atosiban are well proven at premature gestations. ${ }^{7,8}$ When this combination therapy is indicated it should be administered, as it improves the medical care of the mother and child. We never stated otherwise! We reiterate that "Preeclamptic patients treated with tocolytics and corticosteroids may be at higher risk for the development of acute pulmonary oedema. Routine diagnostic echocardiography and lung ultrasound scanning; and continuous monitoring of arterial oxygen saturation with pulse oximetry, should be considered in these patients. ${ }^{2}$ We feel confident that, when the combination of corticosteroid and tocolytic is administered to a preeclamptic patient, cardiopulmonary monitoring is of great (diagnostic) support in dealing with an acute deterioration in maternal and fetal health.
K. Nijs

Department of Anesthesiology and Pain Medicine Jessa Hospital, Hasselt, Belgium E-mail address: kristof.nijs@hotmail.com

K. Nulens

Department of Gynecology and Obstetrics University Hospitals Leuven, Leuven, Belgium

J. Dubois

Department of Anesthesiology and Pain Medicine Jessa Hospital, Hasselt, Belgium

M. Van de Velde

Department of Anesthesiology and Pain Medicine University Hospitals Leuven, Leuven, Belgium

B. Stessel

Department of Anesthesiology and Pain Medicine Jessa Hospital, Hasselt, Belgium

Limburg Clinical Research Program, Hasselt University and Jessa Hospital, Belgium

Department of Anesthesiology, Maastricht University Medical Center+, Maastricht, the Netherlands

\section{References}

1. Guy J, O'Kane E, Eggleton A. Steroids, atosiban and pulmonary oedema; are or may be a cause? Int J Obstet Anesth 2018;34:115.

2. Nijs K, Nulens K, Dubois J, Van de Velde M, Stessel B. The combination of corticosteroid and tocolytic therapy in a preeclamptic patient is a risk factor for the development of acute pulmonary oedema. Int J Obstet Anesth 2018;34:113-4.

3. Dennis AT, Solnordal CB. Acute pulmonary oedema in pregnant women. Anaesthesia 2012:67:646-59.

4. Available at: https://www.medicines.org.uk/emc/product/3262 [Accessed January 2018].

5. Fernandez A, Dominguez D, Delgado L. Severe non-cardiogenic pulmonary oedema secondary to atosiban and steroids. Int $J$ Obstet Anesth 2011:20:189-90.

6. Kurjak A, Chervenak FA. Textbook of Perinatal Medicine. $3^{\text {rd }}$ ed. JP Medical Ltd; 2015. p. 2314.

7. Amorim MM, Santos LC, Faundes A. Corticosteroid therapy for prevention of respiratory distress syndrome in severe preeclampsia. Am J Obstet Gynecol 1999;180:1283-8.

8. Roberts D, Brown J, Medley N, Dalziel SR. Antenatal corticosteroids for accelerating fetal lung maturation for women at risk of preterm birth. Cochrane Database Syst Rev 2017:3:CD004454.

0959-289X/\$ - see front matter

2018 Elsevier Ltd. All rights reserved.

https://doi.org/10.1016/j.ijoa.2018.02.006

\section{Intrathecal tranexamic acid - an} accident waiting to happen?

A clinical trial has established the safety and efficacy of tranexamic acid in the management of obstetric haemorrhage. ${ }^{1}$ Although this study was looking at the treatment of haemorrhage, some units are already using 